\title{
LE SCELTE DI UN GENTILUOMO. IL PERCORSO SCIENTIFICO DI EMILIO GABBA ATTRAVERSO LE SUE RACCOLTE DI SCRITTI
}

\author{
JEAN-LOUIS FERRARY $(*)$
}

SunTO. - Emilio Gabba prediligeva gli articoli per esprimere i risultati della sua ricerca. L'esame delle dieci raccolte, promosse dallo stesso Gabba, in cui questi articoli sono stati di volta in volta riuniti permette non solo di mostrare le linee di forza della sua ricerca, ma fornisce anche un indice del modo in cui Emilio Gabba ha individuato i principali assi della sua ricerca e li ha sviluppati nel tempo.

$* * *$

ABSTRACT. - An examination of the ten collections, promoted by Emilio Gabba himself, in which his articles have been reunited, not only allows us to show the lines of strength of his research, but also furnish an indication of the choices through which the author had identified the major axes of his own research and the changes they underwent over time.

Desidero, prima di tutto, esprimere la mia profonda riconoscenza all'Istituto Lombardo per avermi invitato una seconda volta a prendere la parola in una delle adunanze, e in modo particolare per avermi proposto di intervenire per rendere omaggio al compianto Emilio Gabba. La mia gratitudine è tanto maggiore in quanto per una ragione di salute non ho potuto prendere parte alla commemorazione che si è svolta a Pavia lo scorso anno e non ho perciò potuto manifestare il rispetto e l'affetto che provo per quel grande studioso che mi ha onorato della sua amicizia. Ho conosciuto Emilio Gabba attraverso i suoi

(*) Istituto Lombardo Accademia di Scienze e Lettere, Milano, Italia. Institut de France - École Pratique des Hautes Études (PSL), France.

E-mail: jean-louis.ferrary@univ-paris1.fr 
scritti prima di incontrarlo - la prima volta fu nel 1975 - in occasione di diversi convegni. Ma è la comune partecipazione a due grandi imprese scientifiche (i Roman Statutes coordinati da Michael Crawford e i lavori del Cedant promossi da Dario Mantovani) che mi ha veramente permesso di conoscere l'uomo, e di apprezzare le sue immense qualità di cortesia venata spesso di una fine ironia, di attenzione, di pazienza e di fedeltà. Ricordo con emozione d'avere ricevuto dopo molti anni una lettera che cominciava con queste parole: "Caro Ferrary, possiamo darci del tu". Niente a che vedere con certe abitudini recenti, che mescolano un po' di demagogia all'imitazione di modi di fare americani, che vogliono che si usi il nome di battesimo e ci si dia del tu quando appena appena ci si conosce. Queste parole di Gabba assumevano per me un senso del tutto diverso, un po' come l'intronizzazione in una confraternita o il conferimento del titolo di cavaliere, perché Gabba, più di ogni altro, incarnava veramente ai miei occhi quello che poteva essere un gentiluomo nel mondo accademico e universitario. E quando ho letto con passione il bellissimo colloquiointervista edito nel 2009 da Umberto Laffi (Emilio Gabba. Conversazione sulla storia) vi ho ritrovato, nella lettera di raccomandazione scritta nel 1958 da Plinio Fraccaro al Rettore dell'Università di Pisa, proprio la stessa caratterizzazione: "è un gentil uomo".

Questo pomeriggio, tuttavia, è meno all'uomo e più allo studioso che devo rendere omaggio. Un compito impressionante, considerata la mole immensa e l'eterogeneità dell'opera (814 pubblicazioni fra il 1949 e il 2006, secondo le due bibliografie esaustive che dobbiamo a Anselmo Baroni e a Donatella Zoroddu), comprese le ricche recensioni e segnalazioni che manifestano l'infaticabile curiosità di Gabba, e di cui non smise mai di arricchire Athenaeum, ma anche la Rivista storica italiana e la Rivista di filologia e d'istruzione classica. Compito spaventevole anche perché ci si può chiedere che cosa resti da dire dopo le celebrazioni che si sono tenute a Pavia e all'Accademia dei Lincei, nell'ambito delle quali le voci più competenti hanno analizzato l'apporto di Gabba ai grandi filoni di ricerca dove s'è maggiormente distinto.

È un taglio un po' diverso quello che vorrei proporvi questo pomeriggio. La bibliografia di Gabba conta una dozzina di monografie o di curatele, alcune delle quali in collaborazione con altri studiosi. $\mathrm{Ma}$ l'essenziale della sua opera (a partire dal 1949, quando Gabba aveva appena 22 anni) è apparsa in primo luogo in riviste, poi in misura minore in volumi di scritti in onore e in atti di convegni, dunque sotto forma 
di articoli (benché a volta lunghi come piccole monografie) o in forma di recensioni. Molti di questi testi successivamente (fra il 1973 e il 2007) sono stati raggruppati da Gabba stesso in dieci raccolte, ciascuna delle quali reca un titolo autonomo (a differenza, per esempio, dei Contributi di Arnaldo Momigliano). Intendo perciò interrogarmi sul significato di queste collezioni di studi, su che cosa ci insegnano dell'evoluzione degli interessi di Gabba, o sulle ragioni che hanno potuto ritardare la ripubblicazione di questo o quell'articolo o serie di articoli: insomma intendo interessarmi a queste raccolte in quanto volumi ciascuno dei quali dotato di una sua unità e pertinenza. Un'osservazione en passant: Gabba ha volontariamente escluso dalle raccolte gli articoli apparsi in volumi collettivi; non solo i suoi contributi degli anni 1989-1992 alla Storia di Roma pubblicata presso Einaudi (in vari volumi e in particolare nel tomo primo del secondo volume, su "La Repubblica imperiale", di cui fu il coordinatore con Guido Clemente e Filippo Coarelli), ma ha escluso anche i due capitoli, apparsi nel 1989 e 1994, che aveva scritto per la nuova Cambridge Ancient History, e pure il contributo su "Mario e Silla", apparso nel 1972 nella collezione su Aufstieg und Niedergang der römischen Welt. Le diverse raccolte, nell'escludere testi che avevano carattere di sintesi e di stato dell'arte, riuniscono esclusivamente ricerca originali e reazioni originali suscitate dalla lettura delle opere più importanti apparse sui temi che lo interessavano più da vicino.

Esercito e società nella tarda repubblica romana (Firenze, La Nuova Italia, collana «Il pensiero storico», 1973) è la prima raccolta pubblicata da Emilio Gabba. É anche la più voluminosa, 623 pagine, la sola (con Italia Romana) provvista di un indice delle fonti antiche, la sola, infine, che sia stata parzialmente tradotta in una lingua straniera, in inglese, già nel 1976 (Oxford, Blackwell). Raggruppa quindici articoli e sette recensioni pubblicati fra il 1949 e il 1971. Dedicata ai suoi maestri dell'Università di Pavia, prima fra tutti a Plinio Fraccaro, contiene in particolare tutti i primi lavori che vertono sull'origine dell'esercito professionale, scaturiti direttamente dalla Tesi di laurea che Gabba aveva sostenuto nel 1948 avendo come relatore Fraccaro, e che concerneva le distribuzioni agrarie per i veterani dell'età tardo-repubblicana. Ma nella raccolta si ravvisano anche due altri nuclei di ricerca. Da una parte, alle riforme di Silla, e al loro rapporto con i progetti di Druso sono consacrati tre articoli pubblicati nel 1956 e 1964, ma si noterà che rimonta a un'appendice delle "Ricerche sull'esercito professionale" del 1951 il primo lavoro sui senatori sillani. Riducendo (fin dal 1949) la 
portata e novità del dilectus mariano del 107, le ricerche di Gabba sottolineavano viceversa l'importanza di Silla, che per l'uso che fece dell'esercitò segnò veramente «il momento di trapasso verso quella nuova fase della vita dello stato Romano che si concluderà con l'Impero» (ES, p. X). D'altra parte, «le origini della Guerra Sociale e la vita politica romana dopo l'89 a. C.» è il titolo di un articolo ampio e molto importante pubblicato nel 1954 in Athenaeum, che si concludeva con dei lunghi ragionamenti su Sertorio e che ha perciò la sua continuazione, in un certo senso, in uno studio del 1970 dedicato alla penisola iberica, intitolato «Aspetti della lotta di Sesto Pompeo in Spagna». Quest'interessamento per la dimensione italiana della crisi tardorepubblicana è palesemente quel che aveva attratto l'attenzione di Gabba verso le Guerre civili di Appiano, specialmente verso il primo libro. Sotto questo profilo, la raccolta Esercito e società (ed è sufficiente consultare l'indice delle fonti antiche) costituisce un insieme del tutto coerente con i tre altri volumi che Gabba aveva già pubblicato presso il medesimo editore: Appiano e la storia delle guerre civili (1956), e le edizioni dei libri primo e quinto delle Guerre civili nella collana «Biblioteca di studi superiori» (1958 et 1970), il cui apparato di commento rimane tuttora un punto di riferimento indispensabile. Nella sua prefazione a Esercito e società, Gabba sottolinea a giusto titolo la coerenza del volume, che non ha la pretesa di studiare e spiegare in tutti i suoi risvolti la profonda trasformazione politica e sociale conosciuta dallo Stato romano nel periodo che corre dai Gracchi ad Augusto, bensì sceglie di porre l'accento su «la posizione, nella società del tempo, dell'esercito di professione, che si viene affiancando, e poi sostituendo, alla tradizionale milizia cittadina» (p. IX), e adotta questo cambiamento fondamentale (in connessione con le sue cause e le sue conseguenze) come un punto d'osservazione privilegiato. Precisa inoltre che ha scelto di non includere nella raccolta i suoi studi su Dionigi d'Alicarnasso (apparsi fra il 1960 e il 1963) in quanto restavano a margine di questo nucleo centrale (pp. X-XI), benché per il tramite di Dionigi queste indagini abbiano contribuito a chiarire l'annalistica romana sillana e postsillana, e che siano dunque legate alle indagini sul pensiero politico tardo-repubblicano. Questa scelta è significativa e ci ricorda che Gabba, nel 1973, avrebbe potuto scegliere di raccogliere i suoi lavori su Appiano, su Dione Cassio e su Dionigi già pubblicati sotto forma di articoli, e avrebbe potuto mettere in apertura del volume il testo della lezione inaugurale che aveva tenuto a Pisa nel 1958 sugli «Storici greci 
dell'impero romano da Augusto ai Severi», riconosciuto da Arnaldo Momigliano come un autentico testo programmatico di grande ampiez$\mathrm{za}$, e per questa ragione da lui accolto l'anno seguente nella Rivista storica italiana. In Esercito e società Gabba preferì riunire dei lavori che formavano un insieme assolutamente coerente, su una tematica che, se non esaurita, egli aveva certamente esaminato in profondità: era un modo di chiudere il capitolo aperto con la tesi di laurea e di proseguire la ricerca privilegiando altri temi, altre angolature. Precisando infine che alcuni amici l'avevano incoraggiato a ripubblicare questi testi, Gabba fa il nome del solo Ernst Badian «con il quale tante volte sono stato in aperta, vivace e sempre proficua discussione su molti dei problemi considerati in questo libro» (p. XI). In un'aggiunta iniziale alla ripubblicazione dell'articolo sulle origini della guerra sociale, ritorna più ampiamente e con maggiore specificità sulla cortese, ma ferma polemica che in effetti l'opponeva a Badian. Badian s'inseriva in una tradizione fondata da Gelzer e ulteriormente irrigidita da Münzer, che riduceva la vita politica romana a quella della sua classe dirigente, e i conflitti che l'agitavano a delle lotte di fazioni, di "Adelsparteien"; Gabba considerava questa concezione come riduttiva, rifiutava di vedere nei membri dell'aristocrazia senatoria i soli veri attori della vita politica, e credeva al ruolo giocato dalle divergenze di interesse economico nei conflitti fra senatori e cavalieri o nell'emersione della rivendicazione da parte degli alleati italiani della cittadinanza romana. Nel mantenere con fermezza il proprio punto di vista («io riconfermo il mio suggerimento che ampia parte deve aver avuto..., soprattutto nel cambiamento intervenuto dopo i Gracchi nell'atteggiamento alleato... anche lo straordinario sviluppo dell'espansione commerciale degli Italici nelle province» [ES, p. 194 n.], Gabba s'accingeva a fare di questa problematica una delle direttrici della sua ricerca negli anni a venire.

Nell'attesa, Per la storia dell'esercito romano in età imperiale (Bologna, Patròn, 1974), include tre soli articoli, due dei quali erano apparsi fra il 1966 e il 1968, mentre il terzo era allora in corso si pubblicazione. Com'è espressamente indicato nella Premessa, è una sorta di complemento di Esercito e società apparso l'anno precedente, allo stesso modo del volumetto Le rivolte militari romane dal IV secolo a. C. ad Augusto (Firenze, Sansoni, 1975). L'insieme veniva a comporre una sorta di trilogia. Gabba, in realtà, non aveva chiuso con le ricerche sull'esercito romano, ma gli articoli più tardi furono inclusi in raccolte organizzate intorno ad altri temi. 
È solo nel 1988 che apparve un volume davvero comparabile con quello del 1973: Del buon uso della ricchezza. Saggi di storia economica $e$ sociale del mondo antico (Milano, Guerini, collana «Saggi»). Vi sono ricompresi quattordici articoli e cinque recensioni usciti fra il 1962 e il 1988, ma due soltanto anteriori al 1973 (un articolo sul progetto di riforma della fiscalità che Cassio Dione fa pronunciare da Mecenate, e una recensione del convegno organizzato da Claude Nicolet sulle "strutture sociali nell'antichità classica"). Questo libro testimonia dunque soprattutto la produzione scientifica di Gabba nei quattordici anni trascorsi dal suo trasferimento a Pavia. Cronologicamente la tarda repubblica ne resta il baricentro, ma molti articoli sottolineano la necessità di risalire fino alla prima metà del $3^{\circ}$ secolo, «momento essenziale, nel quale si colloca un processo di trasformazione della società romana non meno importante di quello che coinvolse tutto l'assetto socio-economico italico nel secolo successivo» (p. 25). C'è una continuità incontestabile fra questi articoli sulla società romana e quelli raccolti in Esercito e società, ma l'esercito ora non è più il cuore pulsante (non compare che nei suoi rapporti con la fiscalità, e in due relazioni a convegni dove l'autore di Esercito e società era stato invitato a intervenire quale specialista dell'esercito romano). Si costata piuttosto l'incremento della problematica economica, studiata nei suoi legami con le conseguenze della conquista (prospettiva nella quale era già stata affrontata nel testo del 1954 sulle origini della guerra sociale), ma soprattutto con un ethos aristocratico di cui Gabba scruta l'evoluzione attraverso le testimonianze delle fonti. La prefazione indica con chiarezza il metodo che costituisce il vero tratto unificante del volume: «il punto di partenza è stato di norma un testo letterario antico, e più delle volte un testo storico. Forse proprio perché... le problematiche economiche non sono propriamente centrali... nella riflessione storico-politica antica, mi è sempre sembrato che fosse importante valorizzare quegli spunti che permettevano di cogliere taluni atteggiamenti o riflessioni di fronte a tematiche di questo genere. Questi saggi, quindi, sono per lo più interpretazioni e commenti a riflessioni antiche su problemi di storia economico-sociale. Non mi sono prefisso lo scopo di analizzare direttamente fatti o fenomeni storico-economici, soprattutto perché ho dei dubbi sulle metodologie adatte per affrontarli (la creazione di 'modelli' quantitativi o più ancora teorici, dei quali oggi molto si discute, mi lascia incerto)». Il bersaglio preso qui di mira è palesemente Moses Finley con la sua scuola (P. Garnsey, K. Hopkins, R. 
Whittaker), come mostra la recensione di Ancient Slavery and Modern Ideology pubblicata nella raccolta stessa (p. 217-235), e che sarà completata nel 1990 da «Modelli interpretativi nella storiografia antica» (articolo ripreso nel 1995 in Cultura classica e storiografia moderna). Un altro articolo metodologico importante («Per la storia della società romana tardo-repubblicana») risale al 1982, in occasione della presentazione dei tre volumi frutto di un convegno dell'Istituto Gramsci e pubblicati a cura di Andrea Giardina e Aldo Schiavone con il titolo Società romana e produzione schiavistica. A fianco delle riserve formulate su un uso generalizzato del modo di produzione schiavistico, che non consente di prendere in considerazione l'importanza delle differenze regionali, spicca un elogio vibrante dei risultati che si possono ottenere da una autentica collaborazione fra storici e archeologi e l'ironia con la quale viene ricordato l'interesse di lavori come quelli di Julius Beloch e poi di Plinio Fraccaro e della sua scuola, anteriori alla diffusione nell'ambito dell'antichistica dei metodi e degli approcci tipici dell'archeologia preistorica anglosassone.

Fra il 1993 e il 1995, tre raccolte compaiono a ritmo annuale. Italia romana (Como, Bibliotheca di Athenaeum 25, venticinque articoli e tre recensioni scritti fra il 1972 e il 1994, ma un solo articolo anteriore al 1973) è incontestabilmente il volume che s'iscrive più sensibilmente in continuità con Esercito e società (1974) e con il Buon uso della ricchezza (1988). Tuttavia, lo spettro cronologico s'estende, perché si rivolge ulteriormente alle conseguenze della guerra sociale, all'urbanizzazione nel I secolo a.C. e all'Italia nell'epoca imperiale, mentre le ricerche di storia economica s'arricchiscono di parecchi articoli sulla transumanza e la catastazione, completando il volume su Strutture agrarie e allevamento transumante nell'Italia romana (III-I sec. a. C.) pubblicato in collaborazione con Marinella Pasquinucci (Pisa, Giardini, 1979), volume che a sua volta riprendeva tre articoli apparsi fra il 1974 e il 1978. Ma è soprattutto il problema dell'unità dell'Italia antica che assume un'importanza tutta nuova, in una riflessione che inizia con l'articolo del 1978 intitolato appunto «Il problema dell' unità'dell'Italia romana» e posto in apertura del volume, e che prosegue svolgendosi fino all'introduzione del 1994: il risultato è un'analisi molto articolata e ricca di sfumature, che sottolinea l'esistenza - per l'Italia — di uno statuto giuridico privilegiato e di un declino economico rispetto alle province, e inoltre una unificazione giuridica che non impedisce tuttavia un potere centrale piuttosto debole: fino alle riforme di Diocleziano municipi e 
colonie godono di una autonomia più effettiva di quella di cui avevano goduto le città alleate prima della guerra sociale. Proprio quest'autonomia scaturisce un attaccamento alla piccola patria che non permette che sorga e prenda respiro un sentimento nazionale italiano. Riascoltiamo qualche parola di Gabba nella sua Conversazione sulla storia, che rinvia esplicitamente al volume Italia romana: «credo che ci sia stata una formazione unitaria la quale non si è tradotta in un'unità culturale, diciamo pure spirituale e sociale dell'Italia... L'Italia è rimasta... uno stato composto da repubbliche municipali, e, secondo me, questa frammentazione... è sempre stata una dimensione peculiare dell'organizzazione politica dell'Italia romana» (p. 30).

Aspetti culturali dell'imperialismo romano (Firenze, Sansoni, 1993), che riunisce nove articoli e cinque recensioni scritte fra il 1955 e il 1992 (ma solo un articolo e una recensione sono anteriori al 1973), prosegue e sviluppa l'indagine sull'espansione romana che già si poteva scorgere delinearsi in Esercito e società con l'articolo su «Le origini della guerra sociale» nonché in vari pezzi successivi inclusi nel Buon uso della ricchezza: ma l'attenzione di Gabba lascia ora in secondo piano gli aspetti economici per privilegiare gli aspetti culturali, e allo stesso tempo dedica maggiore interesse alle interpretazioni e reazioni dei Greci di fronte alla conquista Romana, di quanto ne riservi all'ideologia dei Romani stessi (anche se bisogna fare eccezione per l'articolo che dà il titolo all'intera raccolta, benché sia anch'esso per buona parte consacrato a Polibio; e un'eccezione ancora più rilevante, per l'attenzione che presta al punto di vista dei Romani, è l'importante articolo «Il consenso popolare alla politica espansionistica romana [III-II secolo a. C.]»). Come nel precedente volume sull'economia, in Aspetti culturali dell' imperialismo romano Gabba sottolinea la fragilità dei modelli interpretativi che pretendono di spiegare tutto, e pérora un ritorno allo studio minuzioso dei testi antichi per quello che ci possono insegnare: «non so, allora, - scrive Gabba - se non valga meglio di interrogare la tradizione antica per scoprire come siano stati intesi, in Grecia e a Roma, i problemi politici e culturali che nascevano dall'espansione romana, il cui momento storicamente più determinante fu senz'altro l'incontro di Roma con il mondo greco. Le reazioni, soprattutto negli aspetti culturali e negli atteggiamenti storiografici, degli ambienti greci, e la ricezione a Roma di tanti motivi della vita culturale e materiale greca sono fatti più significativi, e meglio conoscibili, che non le cause dell'imperialismo romano» (38). 
Come ho ricordato in occasione della recente riedizione (Roma, 2014), il mio libro su Philhellénisme et impérialisme, concepito negli anni Settanta, fu profondamente influenzato dalla lettura di questi lavori di Emilio Gabba (p. 688). Nella recensione che poi ne fece nella Rivista Storica Italiana, pur senza nascondere i dissensi abbastanza numerosi che potevano dividerci sull'interpretazione di questa o quella fonte, Gabba sottolineò il consenso di fondo che ci univa nel modo di affrontare una tematica abbastanza tremenda come l'imperialismo. Ebbi poi il gran piacere di constatare che aveva incluso questa recensione proprio nei suoi Aspetti culturali, di cui costituisce il testo conclusivo.

Cultura classica e storiografia moderna (Bologna, Il Mulino, 1995), raccolta di dodici articoli, sei discussioni critiche e cinque ritratti di storici scritti fra il 1956 e il 1995 (di cui quattro anteriori al 1973), segna incontestabilmente una nuova tappa nel percorso di ricerca di Gabba, perché è il primo volume integralmente consacrato alla storiografia antica («Storia vera e storia falsa nell'antichità classica»), ma soprattutto alla storiografia sull'Antichità, a partire dal diciottesimo secolo. L'interesse di Gabba per questa tematica non ci sorprende; la sua famiglia possedeva un'importante biblioteca il cui primo nucleo era stato costituito nel Settecento e all'inizio dell'Ottocento; inoltre, il giovane Gabba fu ammesso nel 1949-1950 all'Istituto italiano per gli studi storici di Napoli, fondato da Croce e diretto da Federico Chabod; e già dal 1947 aveva conosciuto Arnaldo Momigliano a Pavia, e in seguito contribuirà a farlo venire alla Scuola Normale Superiore di Pisa dal 1964, mentre Momigliano, a sua volta, favorì la sua collaborazione alla Rivista Storica Italiana, poi raccomandò nel 1981 il suo ingresso nel comitato di direzione della rivista a fianco di Franco Venturi. Anche le raccolte anteriori a quella del 1995 sono ricche di testi sulla storiografia antica, ma includono pochi interventi sulla storiografia moderna sull'Antichità, se si eccettuano ovviamente le recensioni (persino l'articolo su «Lo Spirito Santo, il senato romano e Bossuet», pubblicato nel 1985 nella Rivista storica italiana e ripreso in Aspetti culturali dell'imperialismo romano, è di fatto largamente consacrato alla visione di Roma nei testi giudaici e cristiani dell'Antichità). L'articolo più risalente ripubblicato in Cultura classica, «Prosper Mérimée, storico di Roma», apparso nel 1956 nella Rivista storica italiana, si spiega nel contesto dei lavori sulla guerra sociale, poiché le Études sur l'bistoire romaine de Mérimée ricomprendevano in effetti un «Essai sur la guerre sociale»e 
una «Conjuration de Catilina». Il punto è che la produzione di Gabba rivolta alla storiografia sull'Antichità diviene veramente abbondante solo a partire dal 1979. Conviene anche ricordare che nel 1985 a Ann Arbor e nel 1987 all'Accademia Americana di Roma fu invitato a tenere le Jerome Lectures su «Antiquity in the Historical and Political Reflections of the Eighteenth Century» e che il volume cha avrebbe dovuto raccoglierne il testo non è mai apparso: tuttavia il testo di due delle conferenze, corretto e corredato di note, fu pubblicato nella Rivista Storica Italiana nel 1996 e ripreso in Riflessioni storiografiche con il titolo «L'eredità classica nel pensiero di John Adams».

Viceversa, l'invito se possibile ancor più prestigioso di tenere a Berkeley le Sather Lectures nel 1980 ebbe come conseguenza la scrittura e la pubblicazione di Dionigi e la storia di Roma antica, dapprima in inglese (Berkeley, University of California Press, 1991), poi in italiano (Bari, Edipuglia, 1996). La collezione cui Gabba diede il titolo Roma arcaica. Storia e storiografia (Roma, Storia e Letteratura, 2000) è tanto strettamente legata ai lavori su Dionigi di Alicarnasso quanto Esercito e società era legato agli studi su Appiano. La collezione Roma arcaica raccoglie venti articoli e quattro recensioni comparsi fra il 1960 e il 1999. Dieci di questi articoli sono totalmente o in gran parte dedicati a diversi aspetti delle Antichità romane di Dionigi di Alicarnasso, in particolare vi si possono leggere i tre «Studi su Dionigi d'Alicarnasso» apparsi in Athenaeum dal 1960 al 1964, e di cui Gabba nel 1974 aveva sentito il bisogno di giustificare l'assenza in Esercito e società. Il sottotitolo dato alla raccolta è specialmente importante, e perfettamente spiegato nella Premessa: «l'elemento di fondo della mia indagine sta nel cercar di determinare il valore e il significato della tradizione storica letteraria che ci è pervenuta... Da questo punto di vista il problema della storia di Roma arcaica è un problema di storia della storiografia... Io mi rendo perfettamente conto che cercare di capire e di interpretare quello che gli antichi sapevano, o credevano di sapere, attorno alla loro storia più antica è, per così dire, una conoscenza di secondo grado, indiretta, che tuttavia ha il vantaggio di farci intendere su quali fondamenti gli antichi stessi avessero costruito la loro tradizione storica... Questo tipo

$1 \quad$ P. Desideri, «Le Thomas Spencer Jerome Lectures di Ann Arbor 1985», in Ch. Carsana e L. Troiani (a cura di), I percorsi di un historikos. In memoria di Emilio Gabba, Como, Biblioteca di Athenaeum 58, 2016 pp. 335-343. 
di indagine... ha in sé un buon grado di ragionevolezza e, oserei dire, di verità e di sicurezza» (p. 7).

Gabba che nel 1982 aveva elogiato i frutti di una collaborazione fra storici e archeologi in rapporto alla storia di Roma medio e tardo repubblicana, non revoca adesso minimamente in dubbio quel principio, ma esprime il suo turbamento di fronte a una tendenza recente che, a proposito delle origini di Roma, invece di confrontare le fonti letterarie e le fonti archeologiche dopo che esse sono state passate al vaglio secondo i metodi e i principi propri di ciascuna delle due serie, cerca invece nei dati archeologici la conferma irrefutabile di una tradizione letteraria che non è stata preventivamente sottoposta a un vero esame critico e metodico (la «Premessa» del volume Roma arcaica in un certo senso preannuncia la recensione con cui il volume stesso si chiude, ossia una lettura molto critica de La nascita di Roma di Andrea Carandini). «Bisogna ribadire qui una convinzione - dice Gabba -: per la storia arcaica di Roma l'analisi storiografica è una premessa indispensabile per la conoscenza di quella storia; è anzi una delle basi stesse di quella storia» (p. 8).

Nello stesso anno 2000, un'altra raccolta riunisce, a proposito della storia di Pavia e della sua Università, quindici articoli pubblicati fra il 1984 e il 1999: Pavia domicilium sapientie. Note storiche (Como, Biblioteca di Athenaeum 46). Dopo il suo ritorno a Pavia da Pisa, nel 1974, Gabba era divenuto (e lo rimase dal 1978 al 2002), presidente della Società Pavese di Storia Patria, e diresse gli otto tomi della Storia di Pavia promossi da questa Società (1984-2000).

Nel 2005 appaiono le Lezioni al Collegio Nuovo (Pavia, Fondazione Sandra e Enea Mattei): dieci testi, sei dei quali erano le versioni semplificate di articoli già pubblicati (fra il 1989 e il 2002), quattro invece totalmente o quasi inediti. Anche se non rappresenta che una parte delle ventidue conferenze tenute immancabilmente con un appuntamento annuo al Collegio Nuovo di Pavia dal 1983 al 2004, questo piccolo libro illustra bene la varietà dei temi che interessavano Gabba, e la sua capacità di esporli anche al di là della cerchia stretta degli specialisti.

L'ultima raccolta di lavori è apparsa nel 2007, con il titolo Riflessioni storiografiche sul mondo antico (Como, Biblioteca di Athenaeum 51), e si presenta dichiaratamente come una prosecuzione del volume Cultura classica e storiografia moderna del 1995. Il rapporto è un po' più complesso. Fra i ventinove testi del volume del 2007, cin- 
que sono anteriori al 1995: in alcuni casi sono articoli che esorbitavano dalla tematica delle precedenti raccolte (in particolare, il bello studio del 1979 «Per un'interpretazione politica del De officiis di Cicerone», oppure quello su «L'Italia dei municipi nell'Impero romano: premesse storiche, politiche e culturali per la storia italiana», che completa la raccolta Italia romana apparsa nello stesso anno 1994); in altri casi si tratta di articoli che non erano stati ripubblicati in precedenza ma che Gabba ha voluto in un certo senso valorizzare includendoli nel volume del 2007 (quelli su Beloch, su Momigliano, sul libro di Mouza Raskolnikoff, Histoire romaine et culture bistorique dans l'Europe des Lumières).

Del pari, si può notare la presenza di un certo numero di ricerche pubblicate dopo il 1995, e che sono incluse in questa collezione benché non attengano in senso stretto alla storiografia antica o alla storiografia moderna sul mondo antico. «Ancora sulle origini di Roma» (2001) è piuttosto un complemento a Roma arcaica, «Roma e la pubblica opinione greca fra il II e il I secolo a. C.» (1999) un complemento a Aspetti culturali dell'imperialismo romano, «La cultura a Pavia negli anni 1773-1805» e «Aspetti della vita universitaria a Pavia alla fine dell'Ottocento» (2001 et 2003) si correlano a Pavia domicilium sapientie.

Per converso, Gabba non ha voluto riprendere studi troppo vecchi, forse perché difficilmente avrebbe potuto ad essi applicarsi il principio enunciato dall'autore nella Premessa: «è sembrato inutile provvedere ad un aggiornamento bibliografico» (p. 21). Testi storiografici importanti, come gli «Studi su Filarco» del 1957 o la lezione inaugurale pisana del $1959 \mathrm{su}$ «Storici greci dell'impero romano da Augusto ai Severi» rimangano così esclusi da tutte le raccolte assemblate dal maestro in vita, e ci sarebbe ampiamente materiale per un volume postumo, anche senza pretendere di compiere la riedizione esaustiva di tutti articoli.

Ad ogni modo, il ricco volume del 2007, che si leggerà tenendo presente gli atti editi da Paolo Desideri e Maria Antonietta Giua, della presentazione che ne è stata fatta a Firenze il 15 ottobre 2009 (Emilio Gabba fra storia e storiografia sul mondo antico, Quaderni della Rivista storica italiana 4, 2011), - dicevo - il ricco volume del 2007 anche se conferma che Gabba ha mantenuto un interessamento costante per $i$ temi che aveva affrontato con maggiore frequenza, mostra non di meno la crescente importanza assunta dalle ricerche di taglio storiografico nell'ultimo decennio della sua produzione.

Che cosa ci ha mostrato questo tragitto attraverso le raccolte dei 
lavori che Emilio Gabba ha composto fra il 1973 e il 2007? Che queste raccolte sono state costituite metodicamente, con una effettiva preoccupazione di coerenza tematica per ciascuna di esse; che esse rivelano al tempo stesso la tastiera estesa degli interessi di Gabba e l'esistenza di 'fils rouges' che percorrono l'opera intera e ne fanno risaltare curve e accentuazioni (così, per esempio, il tema dell'Italia è nato da una riflessione sulle origini della guerra sociale, che si iscrive dapprima nel quadro generale di una duplice riflessione sull'esercito e la società e sui fattori economici della conquista, finché l'accento vien posto da ultimo sui limiti in cui si possa parlare di unificazione dell'Italia romana). Ma la coerenza dell'opera, non meno che nei temi affrontati, mi pare consistere nel metodo. Gabba diffida di modelli prefabbricati, e quel che lo interessa, quel che gli pare un terreno più solido, sono i testi, le fonti antiche che occorre sempre contestualizzare, interpretare, commentare, sottomettendolo a un metodo critico esigente; e altrettanto vale per la storiografia moderna sull'Antichità, che bisogna essa pure contestualizzare, e che a quel punto ci permette di arricchire la nostra stessa riflessione sulle fonti antiche. Per concludere, vorrei lasciare ancora una volta la parola a Emilio Gabba stesso, nella sua Conversazione sulla storia: «per l'utilizzazione corretta di ogni testo letterario antico, storiografico e non, è preliminarmente indispensabile la determinazione, quando sia possibile, del pubblico al quale l'autore si rivolge. Si deve inoltre valutare cosa i lettori antichi leggessero, e, per l'oratoria, cosa potessero comprendere delle argomentazioni politiche, religiose e narrative... Ripeto, a me interessa capire perché gli antichi dicessero certe cose e a che pubblico si rivolgessero. Questa convinzione è venuta sempre più amplificandosi» (pp. 32-34). 